\title{
Characterisation of rapid progressors to type 1 diabetes among children with HLA-conferred disease susceptibility
}

\author{
Petra M. Pöllänen ${ }^{1,2}$ • Johanna Lempainen ${ }^{3}$ • Antti-Pekka Laine ${ }^{3}$. Jorma Toppari ${ }^{4}$. \\ Riitta Veijola $^{5}$ - Paula Vähäsalo ${ }^{5}$. Jorma Ilonen ${ }^{3}$ - Heli Siljander ${ }^{1,2}$ • Mikael Knip ${ }^{1,2,6,7}$
}

Received: 30 May 2016 / Accepted: 17 February 2017 /Published online: 31 March 2017

(C) Springer-Verlag Berlin Heidelberg 2017

\begin{abstract}
Aims/hypothesis In this study, we aimed to characterise rapid progressors to type 1 diabetes among children recruited from the general population, on the basis of HLA-conferred disease susceptibility.

Methods We monitored 7410 HLA-predisposed children participating in the Finnish Type 1 Diabetes Prediction and Prevention (DIPP) study for the development of beta cell autoimmunity and type 1 diabetes from birth over a median follow-up time of 16.2 years (range 0.9-21.1 years). Islet cell antibodies (ICA) and autoantibodies to insulin (IAA), GAD (GADA) and islet antigen 2 (IA-2A) were assessed as markers
\end{abstract}

Heli Siljander and Mikael Knip are joint senior authors.

Electronic supplementary material The online version of this article (doi:10.1007/s00125-017-4258-7) contains peer-reviewed but unedited supplementary material, which is available to authorised users.

Mikael Knip

mikael.knip@helsinki.fi

1 Children's Hospital, University of Helsinki and Helsinki University Hospital, P.O. Box 22, FI-00014 Helsinki, Finland

2 Research Programs Unit, Diabetes and Obesity, University of Helsinki, Helsinki, Finland

3 Immunogenetics Laboratory, University of Turku and Turku University Hospital, Turku, Finland

4 Department of Pediatrics, University of Turku and Turku University Hospital, Turku, Finland

5 Department of Pediatrics, PEDEGO Research Group, Medical Research Center, Oulu University Hospital and University of Oulu, Oulu, Finland

6 Tampere Center for Child Health Research, Tampere University Hospital, Tampere, Finland

7 Folkhälsan Research Center, Helsinki, Finland of beta cell autoimmunity. Rapid progression was defined as progression to clinical type 1 diabetes within 1.5 years of autoantibody seroconversion. We analysed the association between rapid progression and demographic and autoantibody characteristics as well as genetic markers, including 25 nonHLA SNPs predisposing to type 1 diabetes.

Results Altogether, 1550 children (21\%) tested positive for at least one diabetes-associated autoantibody in at least two samples, and $248(16 \%)$ of seroconverters progressed to type 1 diabetes by the end of 2015. The median time from seroconversion to diagnosis was 0.51 years in rapid progressors $(n=42,17 \%)$ and 5.4 years in slower progressors. Rapid progression was observed both among young $(<5$ years) and early pubertal children ( $>7$ years), resulting in a double-peak distribution of seroconversion age. Compared with slower progressors, rapid progressors had a higher frequency of positivity for multiple $(\geq 2)$ autoantibodies and had higher titres of ICA, IAA and IA-2A at seroconversion, and there was a higher prevalence of the secretor genotype in the FUT2 gene among those carrying the high-risk HLA genotype. Compared with autoantibody-positive non-progressors, rapid progressors were younger, were more likely to carry the high-risk HLA genotype and a predisposing SNP in the PTPN22 gene, had higher frequency of ICA, IAA, GADA and IA-2A positivity and multipositivity, and had higher titres of all four autoantibodies at seroconversion.

Conclusions/interpretation At seroconversion, individuals with rapid progression to type 1 diabetes were characterised by a younger age, higher autoantibody titres, positivity for multiple autoantibodies and higher prevalence of a FUT2 SNP. The double-peak profile for seroconversion age among the rapid progressors demonstrates for the first time that rapid progression may take place not only in young children but also in children in early puberty. Rapid progressors might benefit from careful clinical follow-up and early preventive measures. 
Keywords Children · Diabetes-associated autoantibodies . GAD antibodies $\cdot$ HLA $\cdot$ IA-2 antibodies $\cdot$ Insulin autoantibodies $\cdot$ Islet cell antibodies $\cdot$ Prediction $\cdot$ Prevention · Type 1 diabetes

$\begin{array}{ll}\text { Abbreviations } \\ \text { DIPP } & \text { Type 1 Diabetes Prediction and Prevention } \\ \text { FUT2 } & 1,2-\alpha \text {-Fucosyltransferase } \\ \text { GADA } & \text { GAD autoantibodies } \\ \text { IA-2 } & \text { Islet antigen 2 } \\ \text { IA-2A } & \text { IA-2 autoantibodies } \\ \text { IAA } & \text { Insulin autoantibodies } \\ \text { ICA } & \text { Islet cell antibodies } \\ \text { JDFU } & \text { JDF units } \\ \text { RU } & \text { Relative units }\end{array}$

\section{Introduction}

Type 1 diabetes is one of the most common chronic autoimmune diseases in children and adolescents. It is characterised by immune-mediated destruction of pancreatic insulinproducing beta cells, eventually leading to total insulin deficiency. Type 1 diabetes is preceded by an asymptomatic prediabetic period, during which autoantibodies against beta cell structures can be detected in the peripheral circulation [1]. Prediabetic autoantibodies are considered to reflect ongoing islet-specific immune activity and are thus suitable for disease prediction. Previous observations in prediabetic individuals have shown that the duration of the preclinical period is highly variable, ranging from only a few months to more than two decades [2]. The variable duration of the preclinical phase suggests that genetic and immunological characteristics and environmental factors might differ between individuals with rapidly and slowly progressing forms of beta cell autoimmunity.

Earlier studies of prediabetic beta cell autoimmunity and progression to clinical diabetes found that individuals at an increased disease risk can be identified based on risk-associated HLA genotypes together with islet-specific autoantibodies in their peripheral circulation $[3,4]$. However, the timescale for progression to clinical type 1 diabetes and the factors influencing the progression rate remain relatively poorly characterised. In the German BABYDIAB study characterising rapid vs slow progression to type 1 diabetes in multiple autoantibody-positive children, no significant differences were detected in prediabetic autoantibody profiles or HLA predisposition between rapid and slow progressors, except for delayed development of IA-2A in slow progressors. In addition, some differences in the distribution of non-HLA alleles were reported [5].

This prospective study describes the demographic, genetic and immunological characteristics of prediabetic beta cell autoimmunity associated with rapid progression to type 1 diabetes in children with HLA-conferred disease susceptibility who were recruited from the general population.

\section{Methods}

Study participants and their samples The Finnish Type 1 Diabetes Prediction and Prevention (DIPP) study is an ongoing longitudinal observational birth cohort study involving three Finnish university hospitals. The objective of the DIPP study is to monitor the appearance of prediabetic islet autoimmunity in children with HLA-defined disease predisposition and identify interventions for delaying or preventing clinical type 1 diabetes in at-risk individuals. Recruitment began in Turku University Hospital in 1994, in Oulu University Hospital in 1995 and in Tampere University Hospital in 1997. The three hospitals cover a population of approximately 1.4 million (26\% of the total population in Finland), and the number of annual births in all three hospitals is around 14,000 ( $24 \%$ of the total number of annual births in Finland).

The local ethical committees of participating hospitals approved the DIPP study protocol. The study has been carried out in accordance with the principles of the Declaration of Helsinki. The legal representatives of all newborn infants gave written informed consent for cord blood samples to be taken for HLA genotyping. Infants with severe congenital abnormalities or disease were excluded from the study, as were families sharing no common language with the study personnel (i.e. Finnish, Swedish or English). Infants with eligible $H L A-D R / D Q$ risk genotypes were invited to participate in immunological surveillance and clinical follow-up, which started at the age of 3 months [6].

Immunological follow-up was performed using venous blood samples obtained at regular clinical visits. In Oulu and Tampere hospitals, samples were taken at 3, 6, 12, 18 and 24 months of age and annually thereafter. In Turku hospital, samples were obtained every 3 months from birth until 2 years of age and then once every 6 months. Children presenting with autoantibodies were invited to follow-up visits every 3 months at all three study centres. All serum samples collected from study participants were stored at $-70^{\circ} \mathrm{C}$ until analysis.

We followed 7410 children (52.6\% boys) with HLA predisposition to type 1 diabetes from birth (Table 1). These children had participated in the DIPP follow-up for at least 1 year before 2003 or had developed clinical type 1 diabetes before the age of 1 year by the end of 2003 (Fig. 1). Thirteen children $(0.2 \%)$ progressed to clinical type 1 diabetes without developing diabetes-associated autoantibodies and were thus excluded from the autoantibody analyses. Twelve of these children had a delay of 3-12 years from the last autoantibody sampling to diagnosis, which probably contributed to the absence of detectable autoantibodies. Autoantibody data for these children were not available at the time of diagnosis, except for 
Table 1 Clinical characteristics: study population

\begin{tabular}{ll}
\hline Characteristic & Median (range) \\
\hline Follow-up time, years & $16.2(0.9-21.1)$ \\
Time from seroconversion to diagnosis, years $^{\text {Age at seroconversion, years }}{ }^{\mathrm{a}}$ & $4.0(0.0-17.0)$ \\
Age at diagnosis, years $^{\text {Autoantibody titre at seroconversion }}{ }^{\mathrm{a}}$ & $5.0(0.2-15.1)$ \\
$\quad$ ICA, JDFU & $7.6(0.9-18.0)$ \\
$\quad$ IAA, RU & $5(3-668)$ \\
GADA, RU & $7.9(3.5-148.7)$ \\
IA-2A, RU & $19.0(5.4-342.1)$ \\
\hline
\end{tabular}

$n=7410$

${ }^{\mathrm{a}}$ Variables used in Cox regression analyses

one child who was autoantibody positive at diagnosis according to the Finnish Paediatric Diabetes Register.

Genetic screening PCR amplification followed by hybridisation with lanthanide-labelled sequence-specific oligonucleotide probes using time-resolved fluorometry was used to genotype cord blood samples for the major type 1 diabetes riskassociated HLA $D R-D Q$ haplotypes [6, 7]. Eligible children carried either the high-risk $H L A-D Q B 1 * 02 / * 03: 02$ genotype or the moderate risk-associated $H L A-D Q B 1 * 03: 02 / x$ genotypes $(x \neq D Q B 1 * 02,03: 01$ or $06: 02)$ [8, 9]. SNPs of 25 non-HLA genes predisposing to type 1 diabetes were analysed to assess associations with rapid disease progression (electronic supplementary material [ESM] Table 1) [10-14]. SNP genotyping was performed using the Sequenom (San Diego, California, USA) platform at the Genome Center of Eastern Finland, University of Eastern Finland, Kuopio, Finland, and was considered successful when the failure rate for the SNP marker analysis was $<10 \%$ [15].

Analysis of diabetes-associated autoantibodies Diabetes-associated autoantibodies were analysed in the Research Laboratory, Department of Pediatrics, University of Oulu, Oulu, Finland. Islet cell antibodies (ICA) were used as the primary screening tool for beta cell autoimmunity until the end of 2002. If a child was ICA positive or progressed to clinical type 1 diabetes during the follow-up, all of his/her prior and subsequent samples were also analysed for insulin

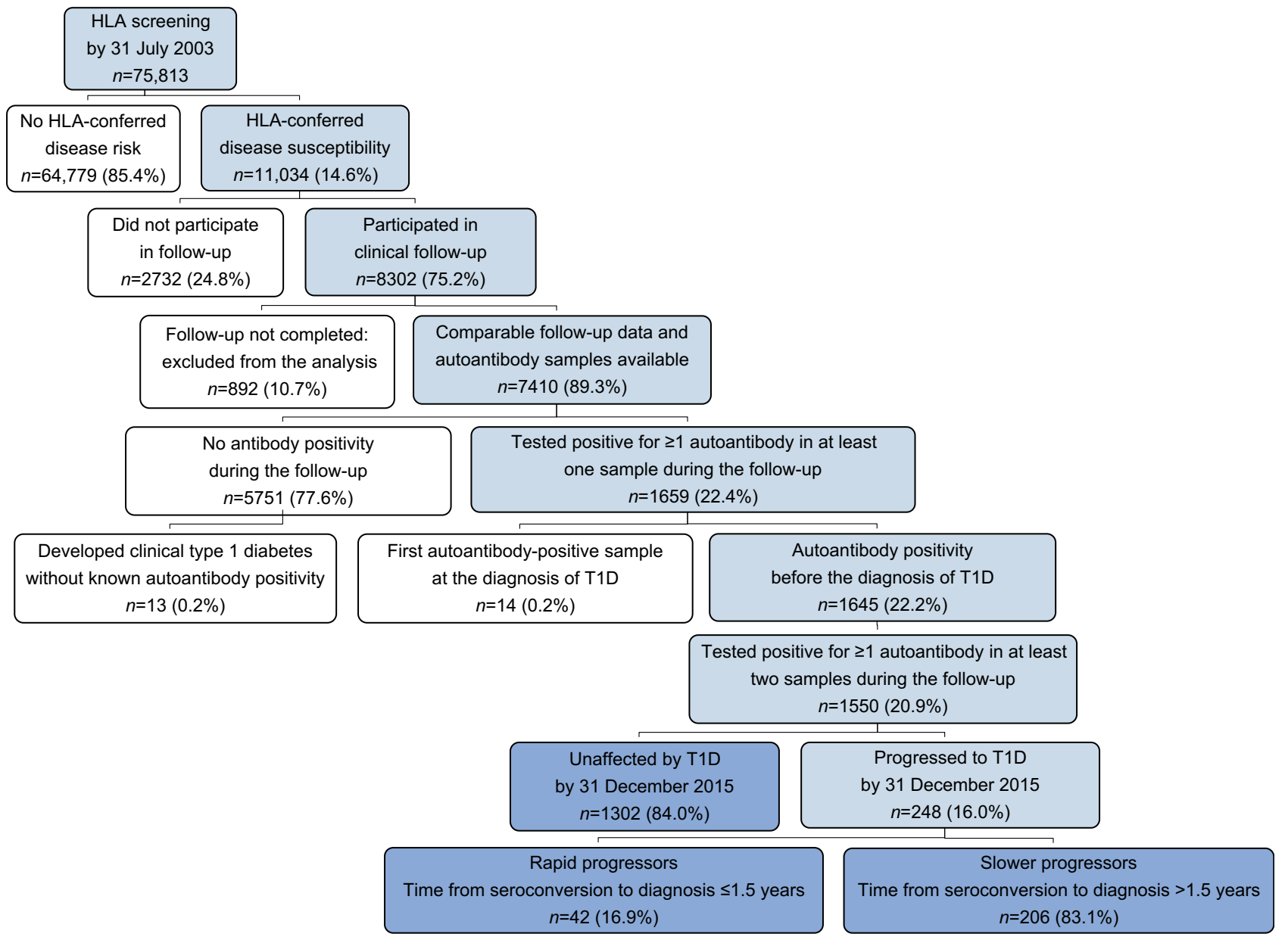

Fig. 1 The DIPP study cohort. T1D, type 1 diabetes 
autoantibodies (IAA), GAD autoantibodies (GADA) and islet antigen 2 autoantibodies (IA-2A). Since 2003, all samples were analysed for ICA, IAA, GADA and IA-2A for study participants born in 2003 and later.

ICA were measured using a standardised indirect immunofluorescence staining method on frozen human pancreas sections from a blood group $\mathrm{O}$ donor, as described previously [16]. The cut-off limit for ICA positivity was 2.5 Juvenile Diabetes Foundation units (JDFU). The biochemical autoantibodies IAA, GADA and IA-2A were analysed with specific radiobinding assays, as previously described [17-19]. Titres of the biochemical autoantibodies were expressed in relative units (RU), representing specific binding of autoantibodies to their cognate antigens. RU were based on a standard curve prepared for each assay plate using MultiCalc software (PerkinElmer Life Sciences-Wallac, Turku, Finland). Cut-off values for positivity were defined as the 99th percentile levels in 370-374 healthy Finnish children: IAA, 3.48 RU; GADA, 5.36 RU; IA-2A, 0.43 RU. All study samples with titres between the 97th and 99.5th percentiles of the reference population values were reanalysed to confirm the result.

According to the Diabetes Autoantibody Standardization Program and Islet Autoantibody Standardization Program workshop results in 2010-2015, disease sensitivities for the IAA, GADA and IA-2A radiobinding assays were 36-62\%, $64-88 \%$ and $62-72 \%$, respectively. The corresponding disease specificities were 94-98\%, 94-99\% and 93-100\%, respectively.

Definitions The time point for seroconversion was defined as the date of the first autoantibody-positive sample. Confirmed autoantibody positivity was defined as positivity for at least one autoantibody in at least two samples. Five progressors diagnosed with classical type 1 diabetes but only one autoantibody-positive sample before diagnosis were considered autoantibody-positive progressors. Multipositivity was defined as positivity for two or more autoantibodies in the same sample. The diagnosis of diabetes was based on WHO criteria [20]. Prediabetes was defined as the detection of diabetes-associated autoantibodies in the peripheral circulation before the diagnosis of clinical type 1 diabetes.

Disease progression was considered to be rapid when type 1 diabetes was diagnosed within 1.5 years of seroconversion to autoantibody positivity. This cut-off limit was partly data driven and partly based on practical considerations: (1) these rapid progressors (17\% of all progressors) represent one of the main targets for future preventive interventions; (2) if the preventive treatment is a success, results become obvious within a short period; and (3) it takes a couple of months to confirm the autoantibody results, exclude overt disease and start preventive treatment. The term 'data driven' indicates that we set out to identify about one-fifth of the study population with the most rapid disease progression.
Data management and statistical analyses Multiple parametric and non-parametric statistical analyses were performed using IBM SPSS Statistics for Macintosh predictive analytics software (version 22.0, Armonk, NY, USA). The CI was set at $95 \%$ and statistical significance was set at $p<0.05$ (twotailed). Cross-tabulation with Pearson's $\chi^{2}$ test and Fisher's exact test, as well as the Mann-Whitney $U$ test or KruskalWallis test, were used to test for significant differences between the groups. Survival distributions according to SNP genotype were tested using the logrank test (Kaplan-Meier analysis). In SNP analyses, Bonferroni correction was used to control for multiple comparisons. Independent variables predicting clinical type 1 diabetes within 1.5 years of seroconversion were tested using the Cox proportional hazards survival model. Group-specific differences in autoantibody levels were analysed only among autoantibody-positive participants (except in Table 2).

\section{Results}

Prevalence of HLA risk genotypes and development of diabetes-associated autoantibodies We followed 7410 HLA-predisposed children from birth for a median of 16.2 years (range 0.9-21.1 years). Altogether, 1550 children (20.9\%) tested positive for at least one autoantibody in at least two samples by the end of $2015 ; 248$ (16.0\%) of autoantibody-positive children progressed to clinical type 1 diabetes. The median age at seroconversion was 5.0 years (Table 1, ESM Fig. 1a). The median time from seroconversion to diagnosis was 0.51 years in rapid progressors ( $n=42,16.9 \%$ of progressors) and 5.37 years in slower progressors ( $n=206$; Table 3, ESM Fig. $1 b)$.

Table 2 Clinical characteristics: young vs older rapid progressors

\begin{tabular}{llll}
\hline Characteristic & \multicolumn{2}{c}{ Rapid progressors } & \multirow{2}{*}{$p$ value } \\
\cline { 2 - 3 } & \multicolumn{1}{c}{ Young $^{\mathrm{a}}(n=36)$} & Older $^{\mathrm{b}}(n=6)$ & \\
\hline Autoantibody titre at seroconversion & & \\
ICA, JDFU & $5(0-320)$ & $49(0-85)$ & 0.15 \\
IAA, RU & $9.5(4.3-66.2)$ & $1.4(0.1-13.0)$ & 0.001 \\
GADA, RU & $5.1(0.1-310.4)$ & $97.7(5.6-158.5)$ & 0.004 \\
IA-2A, RU & $0.11(0.05-108.6)$ & $0.12(0.05-52.51)$ & 0.77 \\
Age at seroconversion, & $1.4(0.3-4.1)$ & $9.0(7.0-13.1)$ & \\
$\quad$ years & & & \\
Age at diagnosis, years & $2.1(0.9-5.5)$ & $9.7(8.2-14.6)$ & \\
$\begin{array}{c}\text { Time from } \\
\text { seroconversion } \\
\text { to diagnosis, years }\end{array}$ & $0.41(0.02-1.45)$ & $0.73(0.59-1.19)$ & \\
\hline
\end{tabular}

Data are medians (range)

${ }^{a}$ Early childhood ( $<5$ years old)

${ }^{\mathrm{b}}$ Early puberty ( $>7$ years old) 
Table 3 Clinical characteristics: rapid progressors vs slower progressors and AAB-positive non-progressors

\begin{tabular}{|c|c|c|c|c|c|c|}
\hline \multirow[t]{2}{*}{ Characteristic } & \multicolumn{3}{|l|}{ Progressors } & \multicolumn{3}{|l|}{ Non-progressors } \\
\hline & Rapid $(n=42)$ & $\begin{array}{l}\text { Slower } \\
(n=206)\end{array}$ & $p$ value $^{\mathrm{a}}$ & $\begin{array}{l}\text { AAB-positive } \\
(n=1302)\end{array}$ & $p$ value ${ }^{\mathrm{b}}$ & $p$ value $^{\mathrm{c}}$ \\
\hline \multicolumn{7}{|l|}{$\mathrm{AAB}$ titre at seroconversion } \\
\hline ICA, JDFU & $34.5(4-320)$ & $10(3-668)$ & 0.001 & $4(3-512)$ & $<0.001$ & $<0.001$ \\
\hline IAA, RU & $10.5(4.3-66.2)$ & $8.8(3.5-81.0)$ & 0.050 & $6.9(3.5-148.7)$ & $<0.001$ & 0.002 \\
\hline GADA, RU & $32.1(5.6-310.4)$ & $28.0(5.8-189.5)$ & 0.51 & $13.5(5.4-342.1)$ & 0.01 & $<0.001$ \\
\hline IA-2A, RU & $52.5(2.6-108.6)$ & $16.4(0.4-121.0)$ & 0.04 & $2.3(0.5-88.9)$ & $<0.001$ & 0.005 \\
\hline Age at seroconversion, years & $1.5(0.3-13.8)$ & $1.9(0.3-12.0)$ & 0.24 & $6.0(0.2-15.1)$ & $<0.001$ & $<0.001$ \\
\hline Age at diagnosis, years & $2.4(0.9-14.6)$ & $8.4(2.1-18.0)$ & & & & \\
\hline Time from seroconversion to diagnosis, years & $0.51(0.02-1.45)$ & $5.37(1.53-17.0)$ & & & & \\
\hline Follow-up time, years & $2.4(0.9-14.6)$ & $8.4(2.1-18.0)$ & & $16.5(12.4-21.1)$ & & \\
\hline
\end{tabular}

Data are medians (range)

${ }^{\text {a }}$ Rapid progressors vs slower progressors

${ }^{\mathrm{b}}$ Rapid progressors vs autoantibody-positive non-progressors

${ }^{\mathrm{c}}$ Slower progressors vs autoantibody-positive non-progressors

AAB, autoantibody

In all, the prevalence at seroconversion was $16.5 \%$ $(n=1224)$ for ICA, $4.1 \%$ for IAA $(n=304), 3.6 \%$ for GADA $(n=264), 1.1 \%$ for IA-2A $(n=84)$, and $2.9 \%$ for multipositivity $(n=213$; Table 4$)$. At seroconversion, 117 children $(1.6 \%)$ were positive for at least two biochemical autoantibodies (IAA, GADA and/or IA-2A). Of the 1224 initially

Table 4 HLA genotype, AAB positivity and disease progression: study population

\begin{tabular}{ll}
\hline Characteristic & $n(\%)$ \\
\hline Sex (male) ${ }^{\mathrm{a}}$ & $3895(52.6)$ \\
HLA genotype $^{\mathrm{a}}$ & \\
$\quad$ High-risk, $D Q B 1 * 02 / * 0302^{\mathrm{b}}$ & $1575(21.3)$ \\
$\quad$ Moderate risk, $D Q B 1 * 0302 / \mathrm{b}^{\mathrm{b}}$ & $5835(78.7)$ \\
$\mathrm{AAB}$ positivity at seroconversion & \\
$\quad \geq 1$ positive AAB in $\geq 2$ samples $^{\mathrm{a}}$ & \\
ICA & $1550(20.9)$ \\
IAA & $1224(16.5)$ \\
$\quad$ GADA & $304(4.1)$ \\
IA-2A & $264(3.6)$ \\
$\quad$ Multipositivity & $84(1.1)$ \\
Progression to clinical type 1 diabetes & $213(2.9)$ \\
During follow-up & \\
Within 1.5 years of seroconversion & $248(16.0)$ \\
\hline
\end{tabular}

$n=7410$

${ }^{\text {a }}$ Variables in the Cox regression analyses

${ }^{\mathrm{b}}$ Where $x \neq D Q B 1 * 02, * 03: 01$ or $* 06: 02$

${ }^{\mathrm{c}}$ Percentage of progressors

$\mathrm{AAB}$, autoantibody
ICA-positive children, 26 (2.1\%) were rapid progressors and $116(9.5 \%)$ were slower progressors, while $60(4.9 \%)$ of those who were initially multipositive and $1022(83.5 \%)$ who were initially single autoantibody positive have not yet progressed to diabetes (non-progressors). Among the 304 initially IAApositive children, 34 (11.2\%) were rapid progressors, 136 (44.7\%) were slower progressors, 37 (12.2\%) are multipositive non-progressors and $97(31.9 \%)$ are single autoantibodypositive non-progressors. Correspondingly, of the 264 initially GADA positive and 84 initially IA-2A positive children, $24(9.1 \%)$ and $11(13.1 \%)$ were classified as rapid progressors, $93(35.2 \%)$ and $43(51.2 \%)$ as slower progressors, $48(18.2 \%)$ and $16(19.0 \%)$ as multipositive non-progressors, and 99 $(37.5 \%)$ and $14(16.7 \%)$ as single autoantibody-positive nonprogressors, respectively.

Rapid vs slower disease development In comparison with slower progressors, rapid progressors had higher titres of ICA, IAA and IA-2A, and a higher frequency of multipositivity at seroconversion (Table 5, ESM Fig. 2). There were no significant differences between groups regarding sex, HLA genotypes, age at seroconversion or positivity for any of the four diabetesassociated autoantibodies (Table 5). Of the diabetes-associated autoantibodies, IA-2A had the highest specificity $(95 \%, n=11)$ and ICA and IAA the highest sensitivities $(62 \%[n=26]$ and $81 \%$ [ $n=34]$, respectively) for rapid disease progression.

Rapid progressors vs autoantibody-positive non-progressors Compared with autoantibody-positive non-progressors, rapid progressors were younger, were more likely to carry the highrisk $H L A-D Q B 1 * 02 / * 03: 02$ genotype, had higher frequencies of 
Table 5 AAB positivity and genotype: rapid progressors vs slower progressors and AAB-positive non-progressors

\begin{tabular}{|c|c|c|c|c|c|c|}
\hline \multirow[t]{2}{*}{ Characteristic } & \multicolumn{3}{|c|}{ Progressors } & \multicolumn{3}{|l|}{ Non-progressors } \\
\hline & $\begin{array}{l}\text { Rapid } \\
(n=42)\end{array}$ & $\begin{array}{l}\text { Slower } \\
(n=206)\end{array}$ & $p$ value $^{\mathrm{a}}$ & $\begin{array}{l}\text { AAB-positive } \\
(n=1302)\end{array}$ & $p$ value & $p$ value $^{\mathrm{c}}$ \\
\hline High-risk HLA genotype & $21(50)$ & $82(40)$ & 0.22 & $319(25)$ & $<0.001$ & $<0.001$ \\
\hline Sex (male) & $25(60)$ & $117(57)$ & 0.75 & $685(53)$ & 0.38 & 0.26 \\
\hline \multicolumn{7}{|l|}{$\mathrm{AAB}$ positivity at seroconversion } \\
\hline ICA & $26(62)$ & $116(56)$ & 0.50 & $1082(83)$ & $<0.001$ & $<0.001$ \\
\hline IAA & $34(81)$ & $136(66)$ & 0.06 & $134(10)$ & $<0.001$ & $<0.001$ \\
\hline GADA & $24(57)$ & $93(45)$ & 0.16 & $147(11)$ & $<0.001$ & $<0.001$ \\
\hline IA-2A & $11(26)$ & $43(21)$ & 0.45 & $30(2)$ & $<0.001$ & $<0.001$ \\
\hline Multipositivity SNP & $30(71)$ & $113(55)$ & 0.048 & $70(5)$ & $<0.001$ & $<0.001$ \\
\hline FUT2 major allele $\mathrm{G}$ homozygosity ${ }^{\mathrm{e}}$ & $13(68)$ & $19(28)$ & $0.03^{\mathrm{d}}$ & $23(31)$ & $0.06^{\mathrm{d}}$ & 0.68 \\
\hline$P T P N 22$ minor allele $\mathrm{A}^{\mathrm{f}}$ & $19(46)$ & $76(38)$ & 0.31 & $167(24)$ & $0.03^{\mathrm{d}}$ & $0.002^{\mathrm{d}}$ \\
\hline
\end{tabular}

Data are $n(\%)$

${ }^{\text {a }}$ Rapid progressors vs slower progressors

${ }^{\mathrm{b}}$ Rapid progressors vs autoantibody-positive non-progressors

${ }^{\mathrm{c}}$ Slower progressors vs autoantibody-positive non-progressors

${ }^{\mathrm{d}}$ Bonferroni corrected $p$ value

${ }^{\mathrm{e}}$ In those with the high-risk HLA genotype

${ }^{\mathrm{f}}$ Some children were not analysed for the PTPN22 polymorphism; for rapid progressors $n=41$, for slower progressors $n=200$ and for AAB-positive non-progressors $n=696$

$\mathrm{AAB}$, autoantibody

ICA, IAA, GADA and IA-2A positivity and multipositivity, higher titres of ICA, IAA, GADA, and IA-2A, and more frequently tested positive for at least two biochemical autoantibodies (IAA, GADA, IA-2A; $48 \%$ vs $2 \% ; p<0.001$ ). There was no significant difference in sex distribution between groups (Table 5). Compared with slower progressors, a smaller proportion of autoantibody-positive non-progressors carried the highrisk HLA genotype (Table $5 ; p<0.001$ ).

\section{Double-peak profile for seroconversion age among rapid} progressors In our study population, rapid progression to type 1 diabetes occurred in two peaks: the first in early childhood and the second in early puberty. The majority of rapid progressors $(n=36)$ developed clinical diabetes before the age of 5 years, while six children were not affected before early puberty (at $8-14$ years). No participants who underwent seroconversion at 5-7 years of age had rapid disease progression. The distinctive distribution profile for seroconversion age was unique to rapid progressors and was not observed in children with slower disease progression (Fig. 2). Compared with slower progressors, rapid progressors were more likely to develop autoantibodies before the age of 1 year and beyond the age of 7 years ( 41 vs $18 \% ; p=0.001)$.

The modest number of participants in the two age groups of rapid progressors resulted in low power for all statistical analyses. However, the frequency of IAA positivity and IAA titre at seroconversion were higher in young vs older rapid progressors
(Tables 2 and 6). Older rapid progressors also had higher rates of GADA positivity and higher GADA titres at seroconversion. Older rapid progressors were predominantly boys $(83 \%)$, although the sex difference was non-significant, probably due to the small numbers of individuals in these subgroups.

Association between non-HLA SNPs and disease progression rate We investigated associations between rapid disease progression and 25 non-HLA SNPs predisposing to type 1

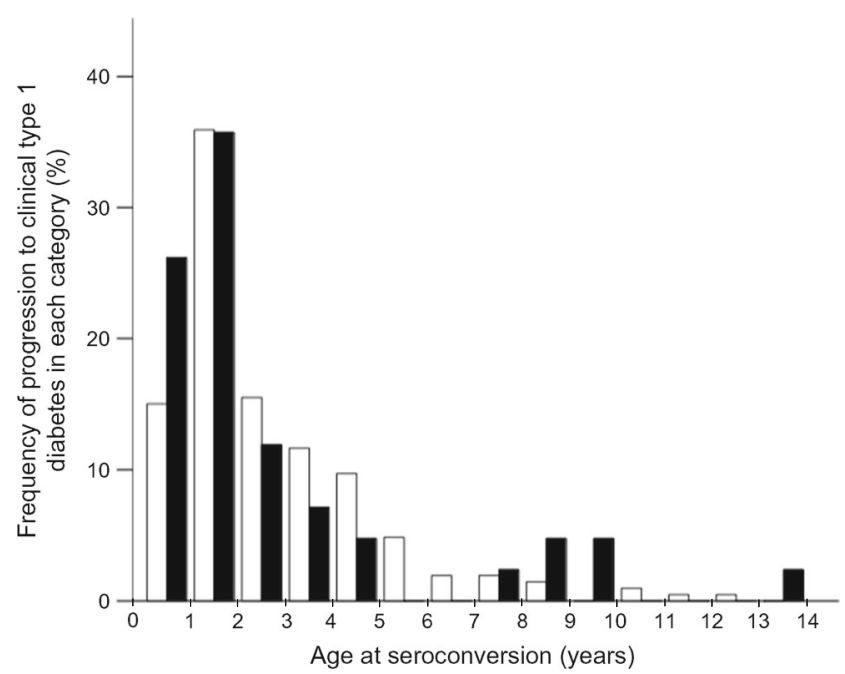

Fig. 2 Age at seroconversion for rapid and slower progressors. Black bars, rapid progressors; white bars, slower progressors 
Table 6 AAB positivity and genotype: young vs older rapid progressors

\begin{tabular}{lrll}
\hline Characteristic & \multicolumn{2}{l}{ Rapid progressors } & \multirow{2}{*}{$p$ value } \\
\cline { 2 - 3 } & $\begin{array}{c}\text { Young } \\
(n=36)\end{array}$ & Older $^{\mathrm{b}}(n=6)$ & \\
\hline Boys & $20(56)$ & $5(83)$ & 0.20 \\
High-risk HLA genotype & $19(53)$ & $2(33)$ & 0.38 \\
AAB positivity at seroconversion & & & \\
$\quad 21(58)$ & $5(83)$ & 0.24 \\
$\quad$ ICA & $32(89)$ & $2(33)$ & 0.001 \\
$\quad 18$ GADA & $18(50)$ & $6(100)$ & 0.02 \\
IA-2A & $9(25)$ & $2(33)$ & 0.67 \\
$\quad$ Multipositivity & $25(69)$ & $5(83)$ & 0.49 \\
FUT2 major allele G homozygosity & $11(65)$ & $2(100)$ & 0.31 \\
\hline
\end{tabular}

Data are $n(\%)$

${ }^{\text {a }}$ Early childhood $(<5$ years old)

${ }^{\mathrm{b}}$ Early puberty ( $>7$ years old)

${ }^{\mathrm{c}}$ In those with the high-risk HLA genotype

$\mathrm{AAB}$, autoantibody

diabetes (ESM Table 1) [15]. After Bonferroni correction, the only significant differences in disease progression were observed in individuals with high-risk HLA genotype. Of these, rapid progressors were more likely to be homozygous for the major $\mathrm{G}$ allele in the FUT2 gene compared with slow progressors (OR 5.70 [95\% CI 1.89, 17.17], corrected $p$ value $p_{c}=0.03$; Table 5) and autoantibody-positive non-progressors (OR 4.90 [95\% CI 1.66, 14.49], $p=0.002, p_{c}=0.06$ ); Table 5). For progressors, the delay from seroconversion to diagnosis was shorter in children with FUT2 major G allele homozygosity than in those with the AG or AA genotype (median 3.0 vs 4.7 years, $p=0.03$ ). In individuals carrying the high-risk HLA genotype and presenting with autoantibodies before the age of 6 years, 34\% of those with the FUT2 GG genotype developed diabetes within 1.5 years of seroconversion, whereas the same was true for only $8 \%$ of those carrying the AG or AA genotype ( $p=0.02$; ESM Fig. 3). Regardless of HLA genotype, a smaller proportion of autoantibody-positive non-progressors carried the PTPN22 minor A allele compared with both rapid progressors (OR 2.74 [95\% CI 1.45, 5.19], $p_{c}=0.03$; Table 5) and slower progressors (OR 1.93 [95\% CI 1.38, 2.70], $\left.p_{c}=0.002\right)$.

Predictive characteristics in a multivariate model Cox regression analysis identified independent variables predicting rapid progression to type 1 diabetes within 1.5 years from seroconversion as the high-risk HLA genotype (HR 1.64 [95\% CI 1.27, 2.12]; $p<0.001$ ), age at seroconversion (HR 0.82 [95\% CI $0.77,0.87]$ ), positivity for multiple autoantibodies (HR 4.32 [95\% CI 2.68, 6.99]), IAA positivity (HR 2.05 [95\% CI 1.35,
3.11]) and the ICA titre at seroconversion (HR $1.003[95 \% \mathrm{CI}$ 1.001, 1.005]; ESM Table 2). FUT2 and PTPN22 SNPs were not included in the model because of missing values. When only statistically significant characteristics found in the first step were included in a subsequent analysis, predictors of rapid progression were identified as the high-risk HLA genotype, positivity for multiple autoantibodies, an ICA titre of $>10$ JDFU, IAA positivity and age at seroconversion (ESM Table 2). ICA positivity was associated with a reduced risk of rapid progression in both analyses, most likely due to the inclusion of biochemical autoantibodies in the analysis.

\section{Discussion}

This longitudinal observational study of 7410 children provides the first characterisation of rapid progressors in children with HLA-conferred disease susceptibility recruited from the general population. We observed that individuals with aggressive islet autoimmunity and rapid progression to type 1 diabetes can be identified from the general population by demographic, genetic and immunological characteristics present at seroconversion. Characterising rapid progression is important because the incidence of type 1 diabetes has been progressively increasing in most Western countries over the past few decades, while age at diagnosis has decreased [21, 22]. This has raised the question of whether the increasing incidence is a result of more aggressive and earlier autoimmune responses in prediabetic individuals [23].

Individuals with rapid disease progression were characterised by a young age, higher prevalence of the high-risk HLA genotype, higher autoantibody titres and a higher rate of positivity for multiple autoantibodies compared with non-progressors. In addition, a higher proportion of rapid progressors carrying the high-risk HLA genotype were homozygous for the major $\mathrm{G}$ allele in the FUT2 gene. Compared with individuals with slower disease progression, rapid progressors had a higher frequency of multipositivity, higher ICA, IAA and IA-2A titres at seroconversion, and a higher frequency of homozygosity for the major $\mathrm{G}$ allele in the FUT2 gene in individuals carrying the high-risk HLA genotype. Sex did not appear to influence the progression rate. The multivariate analysis identified the high-risk HLA genotype, age at seroconversion, multiple autoantibody, ICA and IAA positivity, and high ICA titre at seroconversion as predictors of rapid disease progression. Our results are in accordance with previous reports that multiple autoantibodies at a young age, appearance of IA-2A as the first biochemical autoantibody and high IAA titres in the first IAA-positive sample increase the risk of rapid progression to clinical type 1 diabetes in childhood [24-26].

Earlier studies into progressive beta cell autoimmunity, mainly focused on identifying factors predicting overall progression to clinical diabetes, suggested that high ICA titres 
and an early appearance of IAA are the most sensitive predictors of progressive islet autoimmunity in young children [3, 4, 27-30]. In this study, we used the new approach of investigating factors associated with an increased progression rate in a comparative setting. Several factors reported to predict overall progression to clinical diabetes also, in fact, explain the progression rate, e.g. development of multipositivity at a young age, high ICA and IAA titres at seroconversion, and persistent vs fluctuating IAA [24, 26-29, 31-33].

The comparison of rapid progressors with autoantibodypositive non-progressors was informative because autoantibody-positive non-progressors probably have protective characteristics against the initiated islet autoimmunity. Studying differences between these two groups might reveal natural immunomodulators, pathomechanisms of type 1 diabetes and potential targets for preventive intervention. Furthermore, it has been suggested that all children positive for more than two biochemical autoantibodies will eventually develop clinical type 1 diabetes [24]. Accordingly, a minor subgroup of non-progressors who develop multiple autoantibodies but remain unaffected during follow-up might comprise individuals with ultraslow disease progression who are at risk of developing clinical type 1 diabetes later in life [25]. Although this study involves the longest observation period for prediabetic individuals (from birth to early adulthood), further studies into their fate later in life are needed.

We observed an interesting tendency for rapid progression to occur in two age peaks: early childhood and early puberty. The autoantibody profiles of these two subpopulations are considerably different at seroconversion: young rapid progressors have a high prevalence and high titres of IAA at seroconversion, while those who undergo seroconversion in early puberty are characterised by GADA positivity and high GADA titres at seroconversion. Neither HLA predisposition nor the investigated non-HLA SNPs explain these differences. In particular, the appearance of IAA as the first autoantibody is characteristic of seroconversion at a young age, whereas GADA typically appears later [25]. Owing to the study design that included monitoring older children with longer blood collection intervals, multipositivity at seroconversion may be more common in older children, especially when involving IA-2A positivity because this is usually the last autoantibody to appear and predicts rapid progression to clinical disease [3]. High ICA titres might reflect GADA and/or IA-2A reactivity. The double-peak age distribution and differences in the autoantibody profiles of rapid progressors of various ages raise the following questions: (1) is disease process similar in all forms of aggressive beta cell autoimmunity; (2) are environmental triggers at sensitive stages of development, such as early childhood and early puberty, important; and (3) should preventive interventions be different for different subpopulations? The observation that most of the older rapid progressors had been autoantibody negative less than a year before seroconversion supports the hypothesis that heterogenous events trigger rapid disease progression. In contrast, signs of beta cell autoimmunity leading to slower progression seem to appear at a similar frequency for ages 5-13 years. These findings suggest that in the future the analysis of prediabetic autoantibodies might be targeted to a few selected age points during childhood, with the first autoantibody assessment taking place at age 1 year, and the second closer to puberty, e.g. at age 6 years. The observed differences should, however, be interpreted cautiously because the subgroups were small.

Rapid progressors carrying the high-risk HLA genotype have an increased prevalence of homozygosity for the major $\mathrm{G}$ allele of a SNP within the FUT2 gene, although not the FUT2 SNP predisposing to type 1 diabetes, which is intriguing [15, 34]. The FUT2 gene determines human secretor status by encoding 1,2- $\alpha$-fucosyltransferase (FUT2), an enzyme responsible for the synthesis of soluble ABO histo-blood group antigens, which are present in bodily fluids and on intestinal mucosa [35]. Individuals carrying at least one functional FUT2 allele (major allele $\mathrm{G}$ ) express $\mathrm{ABO}$ antigens in secretions and on the intestinal mucosa (and are thus called secretors), while individuals homozygous for the nonfunctional minor allele A (in populations of European origin) lack the functional enzyme and therefore soluble ABO antigens (and are called non-secretors) [36].

Our results showed that individuals homozygous for the functional FUT2 allele $\mathrm{G}$ are predisposed to rapid disease progression when they carry the high-risk $H L A-D Q B 1 * 02 /$ *03:02 genotype. The association of this FUT2 supersecretor genotype with rapid disease progression suggests that higher levels of soluble ABO antigens in secretions and on the intestinal mucosa might contribute to triggering aggressive beta cell autoimmunity and accelerated disease progression. Gene-gene or gene-environment interactions involving the high-risk HLA genotype may be different in rapid and slower progressors. As an example, the FUT2 SNP is associated with rapid progression only in individuals carrying the high-risk HLA genotype. Further studies are needed to assess the role of genetic and gene-environment interactions in the determination of progression rate.

The strengths of this study are the large, general population-based study cohort and that participants were invited to regular immunological follow-up on the basis of their HLA predisposition. Follow-up started from birth, thus facilitating close collaboration with the families of study participants. Despite the initially large study cohort, there was only a modest number of progressors to overt type 1 diabetes (in particular, rapid progressors), which limited the power of statistical analyses. Since the definition of rapid progression is based on practical considerations and is partly data driven, the generalisability of the results may be limited. However, the findings suggest that 1.5 years after seroconversion is a meaningful cut-off point to discriminate between rapid and slower disease progression. 
To conclude, children with aggressive beta cell autoimmunity and rapid progression to type 1 diabetes may be identified at seroconversion to autoantibody positivity by their demographic, genetic and immunological characteristics including young age, high ICA, IAA, GADA and IA-2A titres, positivity for multiple autoantibodies, and higher prevalence of a FUT2 gene SNP. Such children might benefit from early metabolic surveillance and early treatment to protect them from developing diabetic ketoacidosis. They might benefit from aggressive interventions aimed at delaying and preventing clinical type 1 diabetes. The double-peak age profile in rapid progressors documents for the first time that rapid progression to clinical disease may occur in children at early puberty and not exclusively in children aged under 5 years.

Acknowledgements The authors acknowledge all participants in the DIPP study and physicians, nurses and technicians at the DIPP study centres and laboratories. Some of these data were presented as an abstract at the 51st EASD Annual Meeting, Stockholm, Sweden, 14-18 September 2015 .

Data availability The study data are available on reasonable request from the corresponding author. The data are not publicly available due to the protection of the identity of the study participants and their clinical data.

Funding This work was supported by the following grants: JDRF International (grant nos 4-1998-274, 4-1999-731, 4-2001-435); European Union (grant no. BMH4-CT98-3314); Novo Nordisk Foundation; Academy of Finland (Centre of Excellence in Molecular Systems Immunology and Physiology Research 2012-2017, Decision no. 250114 and grant 292538); Special Research Funds for University Hospitals in Finland; Diabetes Research Foundation, Finland; Sigrid Juselius Foundation; and Finska Läkaresällskapet.

Duality of interest The authors declare that there is no duality of interest associated with this manuscript.

Contribution statement PMP collected and researched the data and substantially contributed to the study conception and design, data acquisition, analysis and interpretation, and drafted the first version of the article. HS substantially contributed to data acquisition, contributed to discussions and edited the manuscript. MK, JL, A-PL, JI, JT, RV and PV generated data, contributed to discussions and revised the article critically for important intellectual content. All authors gave their final approval of the version to be published. MK designed the study, is the guarantor of this work, had full access to all data in the study, and takes responsibility for the data integrity and accuracy of the data analysis.

\section{References}

1. Knip M (2002) Natural course of preclinical type 1 diabetes. Horm Res 57(Suppl 1):6-11

2. Knip M, Korhonen S, Kulmala P et al (2010) Prediction of type 1 diabetes in the general population. Diabetes Care 33:1206-1212

3. Kimpimäki T, Kulmala P, Savola K et al (2002) Natural history of beta-cell autoimmunity in young children with increased genetic susceptibility to type 1 diabetes recruited from the general population. J Clin Endocrinol Metab 87:4572-4579

4. Kukko M, Kimpimäki T, Korhonen S et al (2005) Dynamics of diabetes-associated autoantibodies in young children with human leukocyte antigen-conferred risk of type 1 diabetes recruited from the general population. J Clin Endocrinol Metab 90:2712-2717

5. Achenbach P, Hummel M, Thümer L, Boerschmann H, Höfelmann D, Ziegler AG (2013) Characteristics of rapid vs slow progression to type 1 diabetes in multiple islet autoantibody-positive children. Diabetologia 56:1615-1622

6. Kupila A, Muona P, Simell T et al (2001) Feasibility of genetic and immunological prediction of type 1 diabetes in a population-based birth cohort. Diabetologia 44:290-297

7. Sjöroos M, Iitiä A, Ilonen J et al (1995) Triple-label hybridization assay type-1 diabetes-related HLA-alleles. BioTechniques 18:870 877

8. Nejentsev S, Sjöroos M, Soukka T et al (1999) Population-based genetic screening for the estimation of type 1 diabetes mellitus risk in Finland: selective genotyping of markers in the HLA-DQB1, HLA-DQA1 and HLA-DRB1 loci. Diabet Med 16:985-992

9. Hermann R, Turpeinen H, Laine AP et al (2003) HLA DR-DQencoded genetic determinants of childhood-onset type 1 diabetes in Finland: an analysis of 622 nuclear families. Tissue Antigens 62: 162-169

10. Barrett JC, Clayton DG, Concannon P et al (2009) Genome-wide association study and meta-analysis find that over 40 loci affect risk of type 1 diabetes. Nat Genet 41:703-707

11. Bradfield JP, Qu HQ, Wang K et al (2011) A genome-wide metaanalysis of six type 1 diabetes cohorts identifies multiple associated loci. PLoS Genet 7:e1002293

12. Smyth DJ, Plagnol V, Walker NM et al (2008) Shared and distinct genetic variants in type 1 diabetes and celiac disease. $\mathrm{N}$ Engl J Med 359:2767-2777

13. Hakonarson H, Grant SF, Bradfield JP et al (2007) A genome-wide association study identifies KIAA0350 as a type 1 diabetes gene. Nature 448:591-594

14. Todd JA, Walker NM, Cooper JD et al (2007) Robust associations of four new chromosome regions from genome-wide analyses of type 1 diabetes. Nat Genet 39:857-864

15. Laine AP, Knip M, Ilonen J et al (2013) Transmission disequilibrium analysis of 31 type 1 diabetes susceptibility loci in Finnish families. Tissue Antigens 82:35-42

16. Bottazzo GF, Florin-Christensen A, Doniach D (1974) Islet-cell antibodies in diabetes mellitus with autoimmune polyendocrine deficiencies. Lancet 7892:1279-1283

17. Williams AJ, Bingley PJ, Bonifacio E, Palmer JP, Gale EA (1997) A novel micro-assay for insulin autoantibodies. J Autoimmun 10: 473-478

18. Savola K, Bonifacio E, Sabbah E et al (1998a) Childhood Diabetes in Finland Study Group: IA-2 antibodies - a sensitive marker of IDDM with clinical onset in childhood and adolescence. Diabetologia 41:424-429

19. Savola K, Sabbah E, Kulmala P, Vähäsalo P, Ilonen J, Knip M (1998b) Autoantibodies associated with type 1 diabetes mellitus persist after diagnosis in children. Diabetologia 41:1293-1297

20. WHO Department of Noncommunicable Disease Surveillance (1999) Definition, diagnosis and classification of diabetes mellitus and its complications. Report of a WHO consultation. Part 1: Diagnosis and Classification of Diabetes Mellitus. WHO/NCD/ NCS/99 p. 259

21. Harjutsalo V, Sjöberg L, Tuomilehto J et al (2008) Time trends in the incidence of type 1 diabetes in Finnish children: a cohort study. Lancet 371:1777-1782

22. Patterson CC, Dahlquist GG, Gyürüs E, Green A, Soltész G, EURODIAB Study Group (2009) Incidence trends for childhood type 1 diabetes in Europe during 1989-2003 and predicted new 
cases 2005-20: a multicenter prospective registration study. Lancet 373:2027-2033

23. Ziegler AG, Pflueger M, Winkler C et al (2011) Accelerated progression from islet autoimmunity to diabetes is causing the escalating incidence of type 1 diabetes in young children. J Autoimmun 37:3-7

24. Ziegler AG, Rewers M, Simell O et al (2013) Seroconversion to multiple islet autoantibodies and risk of progression to diabetes in children. JAMA 309:2473-2479

25. Ilonen J, Hammais A, Laine AP et al (2013) Patterns of $\beta$-cell autoantibody appearance and genetic associations during the first years of life. Diabetes 62:3636-3640

26. Parikka V, Näntö-Salonen K, Saarinen M et al (2012) Early seroconversion and rapidly increasing autoantibody concentrations predict prepubertal manifestation of type 1 diabetes in children at genetic risk. Diabetologia 55:1926-1936

27. Bonifacio E, Bingley PJ, Shattock M et al (1990) Quantification of islet-cell antibodies and prediction of insulin-dependent diabetes. Lancet 335:147-149

28. Riley WJ, MacLaren NK, Krischer J et al (1990) A prospective study of the development of diabetes in relatives of patients with insulin-dependent diabetes. N Engl J Med 323:1167-1172

29. Bingley PJ (1996) Interactions of age, islet cell antibodies, insulin autoantibodies, and first-phase insulin response in predicting risk of progression to IDDM in ICA ${ }^{+}$relatives: the ICARUS data set. Islet Cell Antibody Register Users Study Diabetes 45:1720-1728
30. Schatz D, Krischer J, Horne G et al (1994) Islet cell antibodies predict insulin-dependent diabetes in United States school age children as powerfully as in unaffected relatives. J Clin Invest 93:2403-2407

31. Steck AK, Johnson K, Barriga KJ et al (2011) Age of islet autoantibody appearance and mean levels of insulin, but not GAD or IA-2 autoantibodies, predict age of diagnosis of type 1 diabetes: Diabetes Autoimmunity Study in the Young (DAISY). Diabetes Care 34: 1397-1399

32. Steck AK, Vehik K, Bonifacio E et al (2015) Predictors of progression from the appearance of islet autoantibodies to early childhood diabetes: The Environmental Determinants of Diabetes in the Young (TEDDY). Diabetes Care 38:808-813

33. Chmiel R, Giannopoulu EZ, Winkler C, Achenbach P, Ziegler AG, Bonifacio E (2015) Progression from single to multiple islet autoantibodies often occurs soon after seroconversion: implications for early screening. Diabetologia 58:411-413

34. Smyth DJ, Cooper JD, Howson JM et al (2011) FUT2 nonsecretor status links type 1 diabetes susceptibility and resistance to infection. Diabetes 60:3081-3084

35. Yang P, Li HL, Wang CY (2011) FUT2 nonfunctional variant: a 'missing link' between genes and environment in type 1 diabetes? Diabetes 60:2685-2687

36. Ferrer-Admetlla A, Sikora M, Laayouni H et al (2009) A natural history of FUT2 polymorphism in humans. Mol Biol Evol 26: 1993-2003 